\title{
Review of: "Chemotherapy and Targeted Therapy for Patients With Human Epidermal Growth Factor Receptor 2-Negative Metastatic Breast Cancer That is Either Endocrine-Pretreated or Hormone Receptor- Negative: ASCO Guideline Update"
}

\author{
Kelly McCann ${ }^{1}$ \\ 1 University of California, Los Angeles
}

Potential competing interests: The author(s) declared that no potential competing interests exist.

Note: Guideline updates in this manuscript are primarily focused on TNBC. Guidelines for endocrine therapies for metastatic HR+HER2- breast cancer is the focus of a separate review published online July 29, 2021 in the Journal of Clinical OncologyJournal of Clinical Oncology (DOI: 10.1200/JCO.21.01392, PMID: 34324367).

Breast cancer treatments change rapidly

Fortunately for advancing patient care but making clinical oncology practice more difficult, metastatic breast cancer guidelines and reviews are out of date almost as soon as they're published. It's exciting but also daunting. As I explain to my patients, the breast cancer world can flip multiple times a year as the big trials are reported out at our conferences, particularly ESMO (European Society for Medical Oncology), SABCS (San Antonio Breast Cancer Symposium), and the Super Bowl of our people: ASCO (American Association of Clinical Oncology). The past five years have been no exception, with the approval of three CDK4/6 inhibitors and PI3K inhibitor alpelisib in hormone receptor positive $(\mathrm{HR}+)$ HER2-nonamplified (HER2-) metastatic breast cancer setting, multiple HER2-targeted therapies for HER2-amplified (HER2+) breast cancer, PARP inhibitors for patients with pathogenic germline mutations in BRCA1 or BRCA2, and the TROP2 antibody-drug conjugate sacituzumab govitecan for triple negative breast cancer (TNBC). The role of immune checkpoint inhibitors has been less clear, possibly because an optimal biomarker for response has yet to be determined.

Should TNBC and HR+HER2- breast cancers be grouped together? ASCO guidelines for treatment of HER2- breast cancer includes both TNBC and HR+HER2- breast cancer, which of course have radically different biologies. HR+HER2- breast cancers tend to be slower-growing, involve bone metastases first and/or exclusively, and there can be a long disease-free interval of 10+ years from original diagnosis, indicating relatively indolent disease; TNBCs tend to recur within 2-3 years 
from original diagnosis, metastasize to the viscera and brain, and be lethal within about 2 years. Historically, breast cancers were grouped into HER2 + versus HER2- (TNBC and HR+HER2-) for the purpose of cytotoxic chemotherapy trials, so TNBC and HR+HER2- cancers resistant to endocrine therapies remain grouped together in guidelines despite having different prognoses in general. As the number of endocrine therapies and targeted therapies (such as PI3K inhibitors) expands, the prognosis of patients with HR+HER2- breast cancers is improved relative to the relatively poor prognosis of TNBC patients. Many clinical trials exclude HR+HER2- breast cancers because they cannot be assessed by RECIST criteria, though bone-only disease is common in clinical practice and can be quite morbid even if not typically lethal. Prognostic predictions for an individual patient are also confounded by the definition of a HR+ malignancy. A cancer with only $11 \%$ estrogen receptor (ER) expression is not actually driven by estrogen, though it's defined as estrogen receptor positive $(E R+)$.

\section{The ASCO guideline updates for TNBC and HR+HER2- breast cancer}

In this very well-considered and well-written ASCO guideline update, recommendations based on data at the time of publication are as follows:

1. Immune checkpoint inhibitors in combination with chemotherapy (drug unspecified) should be offered as first line therapy for PDL1+ TNBC, but not for PDL1- TNBC.

2. Sacituzumab govitecan can be offered after 2 prior chemotherapies in the metastatic setting for TNBC.

3. PARP inhibitors olaparib or talazoparib may be offered for gBRCA1/2 TNBC after prior treatment with chemotherapy in the (neo)adjuvant or metastatic setting.

4. PARP inhibitors may be offered for HR+HER2- in the metastatic setting to those no longer benefiting from endocrine therapy.

5. Cytotoxic chemotherapy may be offered prior to exhaustion of endocrine therapies for metastatic HR+HER2- breast cancer.

6. In general, single agent therapies should be employed except possibly in life-threatening disease.

\section{Is immunotherapy beneficial in TNBC?}

At the time of publication, the first of the guidelines above was backed by IMpassion130 (NCT02425891), a trial in which nab-paclitaxel was given in combination with atezolizumab or placebo in the first line setting to patients with PDL1+ TNBC, with PDL1 positivity defined as tumor infiltrating lymphocyte (TIL) staining $\geq 1 \%$ of the tumor area using the SP142 antibody [Schmid P, et al. "Atezolizumab and nab-paclitaxel in advanced triple-negative breast cancer." N Engl J Med. 2018; 379: 2108-2121; Schmid P, et al.

“Atezolizumab plus nab-paclitaxel as first-line treatment for unresectable, locally advanced or metastatic triple-negative breast cancer (IMpassion130): updated efficacy results from a randomised, double-blind, placebo-controlled, phase 3 trial." Lancet Oncol. 2020; 21: 44-59.]. The United States Food and Drug Administration (FDA) granted accelerated approval to atezolizumab in combination with nab-paclitaxel for PDL1+ TNBC in March 2019 based on an improvement in progression-free survival and immature overall 
survival data. Approval was contingent on the results of IMpassion131 (NCT03125902) with paclitaxel in combination with atezolizumab versus placebo, which failed to demonstrate a progression-free survival benefit in PDL1+ patients [Miles D, et al. “Primary results from IMpassion131, a double-blind, placebocontrolled, randomised phase III trial of first-line paclitaxel with or without atezolizumab for unresectable locally advanced/metastatic triple-negative breast cancer." Ann Oncol. 2021; 32(8): 994-1004.], which resulted in withdrawal of FDA-approval. Though not statistically significant, ultimately IMpassion130 did show an improvement in overall survival for PDL1+ TNBC patients treated with atezolizumab + nabpaclitaxel versus nab-paclitaxel alone of 25.4 months versus 17.9 months [Emens LA, et al. "First-line atezolizumab plus nab-paclitaxel for unresectable, locally advanced, or metastatic triple-negative breast cancer: IMpassion130 final overall survival analysis." Ann Oncol. 2021; 32(8): 938-993.].

In KEYNOTE-355 (NCT02819518), the chemotherapy options were liberalized to nab-paclitaxel, paclitaxel, or gemcitabine plus carboplatin in combination with pembrolizumab versus placebo in the first line metastatic setting in TNBC. A median progression-free survival benefit was demonstrated in PDL1+ patients, defined as a combined positivity score (CPS) $\geq 10 \%$ of tumor cells, lymphocytes, and macrophages stained for PD-L1 using the 22C3 antibody [Cortes ], et al. "Pembrolizumab plus chemotherapy versus placebo plus chemotherapy for previously untreated locally recurrent inoperable or metastatic triple-negative breast cancer (KEYNOTE-355): a randomised, placebo-controlled, double-blind, phase 3 clinical trial." Lancet. 2020; 396(10265): 1817-1828.]. The FDA granted accelerated approval to pembrolizumab in this setting in November 2020. Accelerated FDA approval was also granted to pembrolizumab in combination with chemotherapy for TNBC in the neoadjuvant setting regardless of PDL1 status based on KEYNOTE-522 (NCT03036488), which highlights the possibility that we don't have the an ideal biomarker.

\section{When should we implement expanded biomarker testing?}

One of the topics the guidelines do not address, but which will become increasingly relevant in the future, is when to add expanded biomarker testing (i.e. beyond ER, PR, and HER2). In cancers such as lung adenocarcinomas, comprehensive biomarker testing is standard-of-care despite the relative rarity of alterations in genes such as $A L K, E R B B 2$, and ROS1. This is not currently the case in breast cancer, though companion diagnostics exist for specific therapies (e.g. PIK3CA mutation testing by PCR for alpelisib eligibility for HR+HER2- breast cancers or PDL1 testing for TNBC). In general, most patients with $\mathrm{HR}+\mathrm{HER} 2$ - breast cancer will respond to endocrine therapy in the first line metastatic setting and most triple negative breast cancers will respond to cytotoxic chemotherapies (albeit temporarily), so the pressure to define disease biology more precisely hasn't been as critical as in rapidly lethal cancers diagnosed at advanced stages and with few treatment options. However, breast cancer is a very common malignancy, and a significant proportion of patients would benefit from identification of targetable driver mutations early on for the purpose of treatment choice as well as prognostic prediction. 
In the case of HR+HER2- breast cancers, if the estrogen pathway is not the oncogenic driver, patients could benefit from earlier implementation of cytotoxic chemotherapy or enrollment in clinical trials prior to exhaustion of endocrine options. For example, a small proportion of women with lobular breast cancers have activating mutations in ERBB2, the gene encoding HER2. Not only would such a patient be eligible for clinical trials targeting HER2, the clinician might also expect more rapid progression on endocrine therapy and consider more frequent follow up as well as earlier implementation of chemotherapy in a type of HR+HER2- cancer traditionally thought to be rather indolent. In clinical practice, I usually order next generation sequencing for cancer mutations at the time of diagnosis of metastatic TNBC and at the time of progression on a non-steroidal aromatase inhibitor in the adjuvant or metastatic setting for HR+HER2cancers to also capture resistance mutations such as activating mutations in ESR1, the gene encoding ER. Activating mutations in ESR1 confer estrogen independence, and the patient would not be an ideal candidate for a treatment such as exemestane plus everolimus.

There is no doubt that the authors of the most recent ASCO guidelines thoroughly appreciate the difficulty of creating recommendations for the rapidly-changing field of treatment options for metastatic HER2breast cancer. The data presented support their recommendations while acknowledging future refinements are expected. 